\title{
Numerical study of emission and dynamics from a TDE-powered jet
}

\author{
P. Mimica ${ }^{1, a}$, D. Giannios ${ }^{2,3}$, B. Metzger ${ }^{3}$, and M.A. Aloy ${ }^{1}$ \\ ${ }^{1}$ Departamento de Astronomia y Astrofisica, Universidad de Valencia \\ 2 Department of Physics, Purdue University, West Lafayette \\ ${ }^{3}$ Department of Astrophysical Sciences, Peyton Hall, Princeton
}

\begin{abstract}
A transient event Swift J1644+57 is thought to be caused by the emission from a collimated relativistic jet. The jet, powered by the sudden onset of accretion onto a supermassive black hole following the tidal disruption of a star, collides with the gaseous circumnuclear medium and produces forward and reverse shocks which emit synchrotron radiation. We perform 1D and 2D relativistic hydrodynamic simulations using the MRGENESIS code. The aim of the simulations is to study the dynamics of a jet thought to exist in transient events such as Swift $\mathrm{J} 1644+57$, as discussed in recent literature. We discuss 1D and 2D jet evolution, on-axis radio light curves and differences between 1D and 2D jet dynamics.
\end{abstract}

\section{INTRODUCTION}

On March 28, 2011 a high energy transient event Swift $\mathrm{J} 1644+57$ was detected, and was soon localized to the centre of a galaxy at redshift $z \simeq 0.353$ [1-3]. A high energy transient event Swift J2058+05 with similar properties was later observed [4], and both of these were argued to result from a rapid increase of the accretion on the supermassive black hole (SMBH) [4-6]. Prior to these observations a possible scenario for the rapid increase of the accretion on the SMBH was explored by Giannios \& Metzger [7], who discuss the observational consequences of a tidal disruption event (TDE) such as a disruption of a massive star by a SMBH [8] and a subsequent launching and acceleration of a collimated jet to relativistic velocities. Because the galactic nucleus is inactive prior to TDE, there is a dense circumnuclear medium (CNM) which quickly decelerates the emerging relativistic jet. The external shocks, which form at the jet-CNM interface, can accelerate particles and produce the observed non-thermal emission $[7,9,10]$.

Metzger, Giannios \& Mimica [9] propose a model for the radio emission from Swift J1644+57. The jet-CNM interaction is assumed to proceed analogously to the GRB afterglow case. There, the jet is assumed to collide with the external medium, which causes the formation of a pair of shocks: forward shock (FS), which propagates into the external medium and a reverse shock (RS), which propagates through the jet [11]. In the particular case of the TDE, it is assumed that the jet kinetic luminosity is not constant, but that its isotropic equivalent follows the law [9]

$$
L_{\mathrm{j}, \text { iso }}(T)=\left\{\begin{array}{ll}
L_{j, 0} & \text { if } T \leq T_{j} \\
L_{j, 0}\left(T / T_{j}\right)^{-5 / 3} & \text { if } T>T_{j}
\end{array},\right.
$$

where $T$ is the time measured in the rest frame of the CNM, $T_{j}$ is the time interval during which the jet luminosity is constant, and $L_{j, 0}$ is the initial jet luminosity. The exponent $-5 / 3$ is obtained by assuming that the jet luminosity is

\footnotetext{
a e-mail: Petar.Mimica@uv.es
}

proportional to the TDE fall-back accretion rate $\dot{M} \propto T^{-5 / 3}$ [8]. In this work we assume $L_{j, 0}=5 \times 10^{47} \mathrm{erg} \mathrm{s}^{-1}$ and $T_{j}=5 \times 10^{5} \mathrm{~s}$. For the CNM we assume that it is cold and that its density declines linearly:

$$
n_{\mathrm{CNM}}(R)=3.33 \times 10^{2}\left(R / R_{j}\right)^{-1} \mathrm{~cm}^{-3},
$$

where $n(R)$ is the CNM number density, $R$ is the distance from the galactic nucleus and $R_{j}$ is the radius at which the jet-CNM interaction begins. Finally, we assume that the jet fluid Lorentz factor is initially $\Gamma_{j}=10$, and that the jet half-opening angle is $\theta_{j}=0.1$ radians.

The motivation for the present work is the need for the assessment of the importance of $2 \mathrm{D}$ effects in the jet evolution. For on-axis models such as the one of [9], we are interested in the effects of the early jet spreading and a possible disruption at later stages. 2D models also allow us to compute off-axis light curves for arbitrary observing angles. Thus we can predict detection rates, power, CNM properties, timescales and frequencies for the events similar to Swift $1644+57$, but oriented arbitrarily with respect to the line of sight.

In Section 2 we discuss the details of the numerical simulations of TDE jets, and in Section 3 we turn our attention to the differences between the 1D and 2D models. Finally, in Section 4 we give preliminary results for the jet emission and summarize our results so far.

\section{NUMERICAL SIMULATIONS OF TDE JETS}

We use the relativistic magnetohydrodynamic code MRGENESIS [12,13] to perform 1D and 2D simulations of the jet-CNM interaction. The jet is injected at point $R_{j}=5 \times 10^{16} \mathrm{~cm}$ and its evolution is followed for 3 years. For 1D simulations we used an equidistant grid of 252000 numerical zones (radial resolution: $10^{13} \mathrm{~cm}$ ), while for the 2D runs we used a $25200 \times 500$ zones (radial resolution: $10^{14} \mathrm{~cm}$, azimuthal resolution: $\pi \times 10^{-3}$ radians). Initially, the jet is resolved using 32 zones in the azimuthal direction.

To compute the synthetic observations from 2D simulations we use the $S P E V$ radiative transfer code 


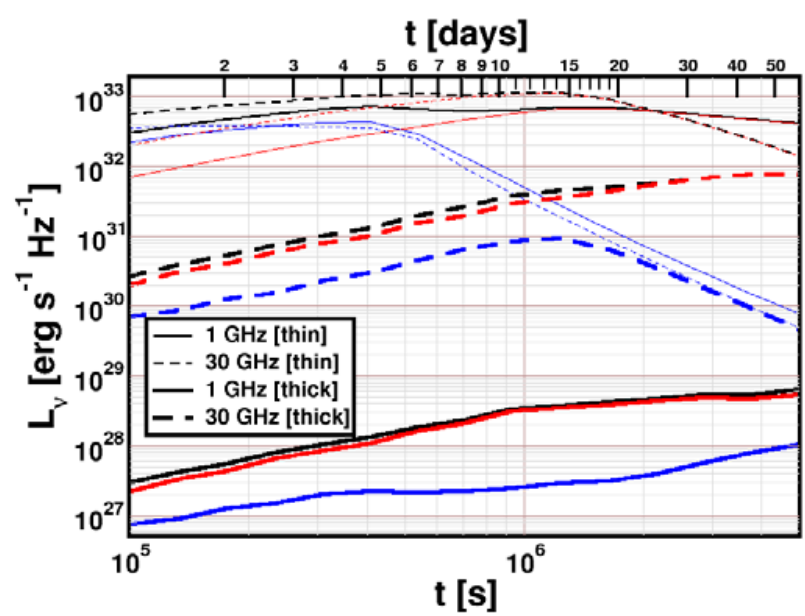

Figure 1. On-axis radio light curves at 1 and $30 \mathrm{GHz}$ (full and dashed lines, respectively) computed from the 1D simulation. Thin and thick lines show light curves assuming optically thin and thick synchrotron emission, respectively. FS and RS light curves are shown in red and black, while the total (FS + RS) light curve is plotted in black.

[14-16]. For 1D simulations we use the modified SPEV scheme which has been used for afterglow light curve calculations [17].

\subsection{D radio light curves and spectra}

As discussed in the previous section, we use the code of [17] to compute the on-axis light curves. In addition to the jet luminosity and geometry we also need to specify the microphysical parameters $\epsilon_{e}, \epsilon_{B}$ and the spectrum of particles injected at relativistic shocks. We use $\epsilon_{e}=0.1$ and $\epsilon_{B}=0.005$, and assume that a power-law distribution $n(\gamma) \propto \gamma^{-p}$ of non-thermal electrons is injected at shocks, where we assume $p=2.3$. See [17] for details on how the lower and upper cutoffs of injected electrons are computed, as well as how the synchrotron and adiabatic losses are treated.

Figure 1 shows the radio light curves at 1 and $30 \mathrm{Ghz}$. To determine the importance of the inclusion of synchrotron self-absorption (SSA), we have computed the light curves both in the optically thin approximation (thin lines) and using the full radiative transfer scheme including the SSA (thick lines). As can be seen, for higher frequency the SSA is not important after $\simeq 40$ days, while for $1 \mathrm{GHz}$ it always needs to be taken into account. This requires substantial computational resources since we need to use the full radiative transfer code instead of a much simpler (and better parallelizable) volume integration scheme used for optically-thin calculations.

A break in (thick) radio light curves can be observed after $\simeq 10$. As discussed in [9,17], this is a consequence of the rarefaction wave crossing the jet and slowing down the fluid behind the FS. The time at which the break is observed is $\simeq 2 T_{j}$ [9].

Figure 2 shows the instantaneous spectra after 10, 20 and 50 days. It can be seen that FS emission always dominates the RS emission. Furthermore, the spectral peak slowly shifts from $\simeq 500 \mathrm{GHz}$ to $\simeq 50 \mathrm{GHz}$ in the time interval considered.

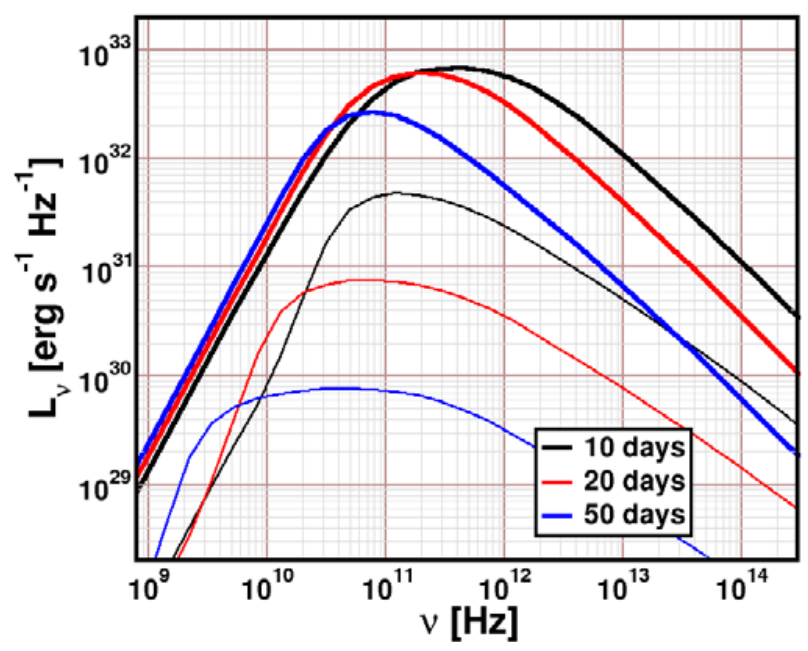

Figure 2. Instantaneous spectra after 10, 20 and 50 days (black, red and blue lines, respectively). Thick and thin lines show the FS and RS contributions.

\subsection{D evolution}

As discussed in Section 1, 2D simulations are used both to check the accuracy of the 1D approximation and to allow us to study off-axis light curves.

As will be discussed in more detail in Section 3, the jet is initially powerful enough to propagate into the $\mathrm{CNM}$, driving the FS into CNM, and is also experiencing deceleration at RS which propagates through the jet. However, due to the decrease of the jet luminosity after $T_{j}$ (see Eq. 1), the rear part of the jet eventually loses support and the cocoon disrupts it. Figure 3 shows a snapshot of the jet evolution $\simeq 193$ days since the beginning of the injection. At this point the jet has already been disrupted by the cocoon, but the FS can still be seen propagating into the CNM. Eventually, FS becomes non-relativistic and enters into the Newtonian phase described by [7].

\section{COMPARISON OF 1D AND 2D MODELS}

In this section we discuss the difference between 1D and 2D simulations. Figure 4 shows the density along the axis for $1 \mathrm{D}$ and 2D models $0.5,0.8,1$ and 1.6 since the start of the jet injection.

As can be seen from the two upper-left panel of Fig. 4, initially $1 \mathrm{D}$ and 2D jets are quite similar, except for a number of density peaks in the 2D model. The structure in front shows three sharp discontinuities, from right to left: FS, contact discontinuity (CD) and RS. The density profile behind the RS has a minimum which is the consequence of the conical jet expansion and the $t^{-5 / 3}$ jet luminosity profile. The density peaks in the $2 \mathrm{D}$ model are located at those places where the cocoon has started to penetrate into the jet. In the upper-right panel it can be seen that the jet behind the RS has been almost completely disrupted, and also the differences in the position of the FS and CD can be seen. In the later stages (lower panels) these differences become ever more visible. This is due to the fact that the disrupted jet transports less energy towards the working surface of the FS and it decelerates faster with respect to the undisrupted $1 \mathrm{D}$ jet. Therefore, it is expected that there 


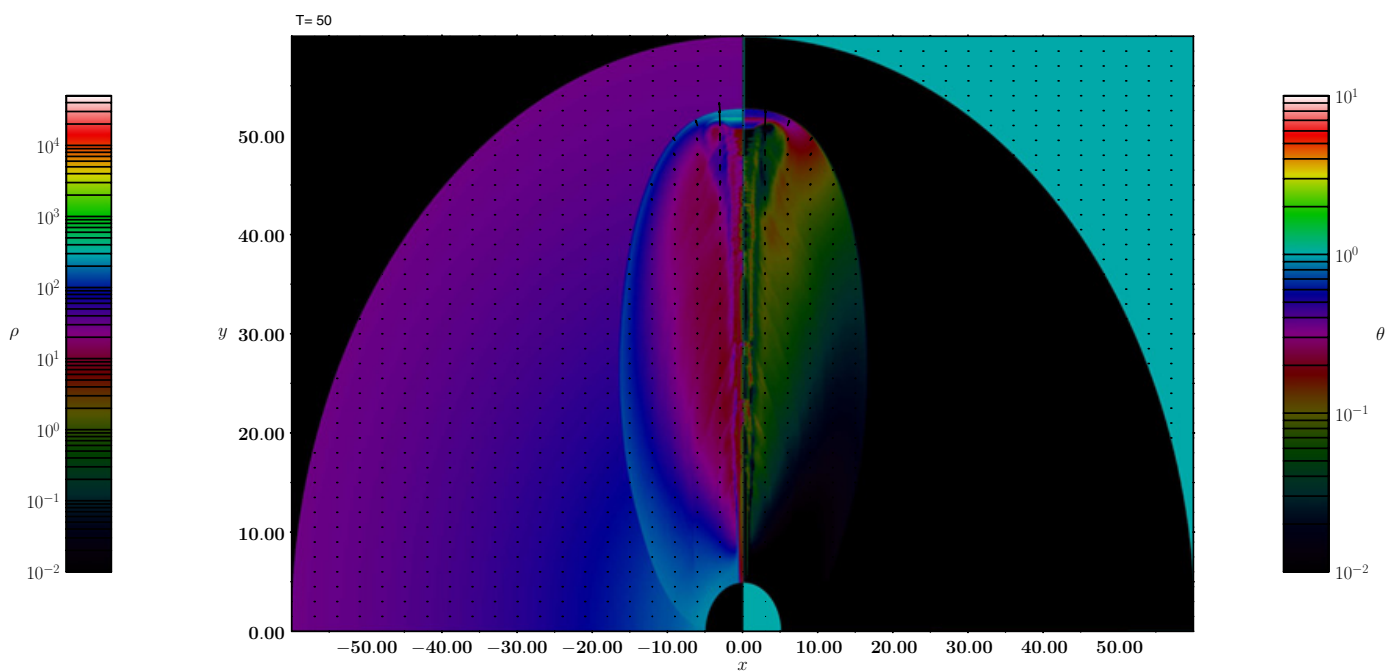

Figure 3. Contours of the density (left panel) and dimensionless temperature parameter $\theta:=P /\left(\rho c^{2}\right)$ (right panel) of the jet $\simeq 193$ days since the beginning of the injection. The unit for density in $\mathrm{cm}^{-3}$, the unit of length is $10^{16} \mathrm{~cm}$, and the unit of time is $10^{16} \mathrm{~cm} / \mathrm{c} \simeq 3.86$ days.
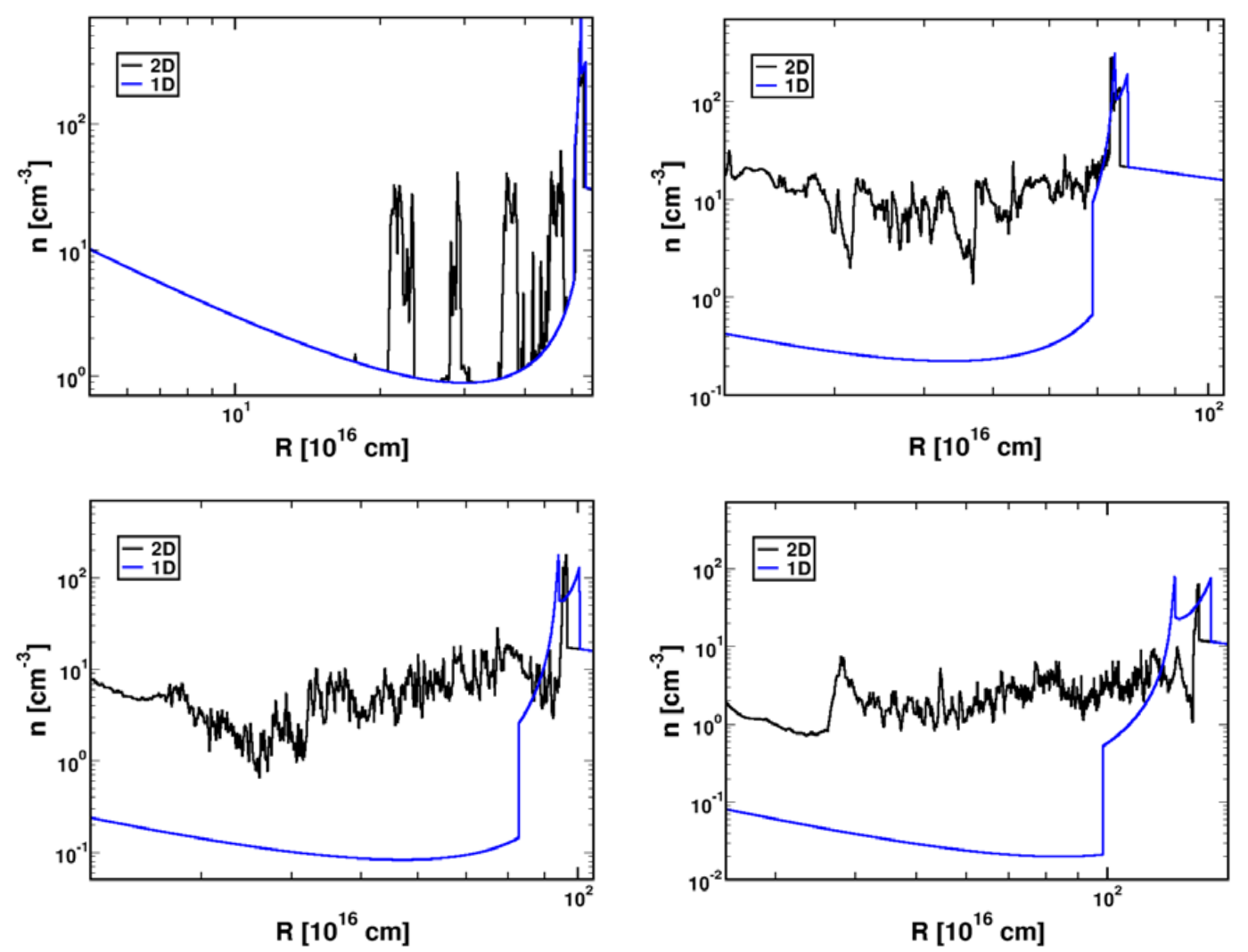

Figure 4. Number density of the fluid along the axis for 1D (blue lines) and 2D (black) simulations 0.5 years (upper-left panel), 0.8 years (upper-right), 1 year (lower-left) and 1.6 years (lower-right panel) after the start of the jet injection.

will be diferences between 1D and 2D models even for the on-axis light curves, especially at later times.

\section{CONCLUSIONS AND FUTURE WORK}

In this article we report on the preliminary results of a project whose aim is to model events such as
Swift J1644+57 in detail using 1D and 2D numerical simulations. We show that the on-axis light curve from 1D models behaves very similarly to the early afterglow light curves of the GRBs: there is an achromatic break in the radio light curve which occurs at $\simeq 2 T_{j}$, and is caused by the rarefaction wave crossing the constant-density part of the jet. We also show that it is important to include the SSA in all calculations, especially for frequencies of $1 \mathrm{GHz}$ and 
lower. 2D simulations show that the jet may get disrupted by the cocoon because of the luminosity dropping as $t^{-5 / 3}$. This has consequences for the late-time propagation of the FS, and will influence the on-axis light curve. Therefore, it is necessary to include the $2 \mathrm{D}$ jet dynamics to get more realistic estimates of long-term radio light curves. In the future work we will show the results of the calculation of the off-axis light curves, and the expected fluxes one can expect for events similar to Swift J1644+57.

PM and MAA acknowledge the support from the European Research Council (grant CAMAP-259276), and the partial support of the Spanish Ministry of Science grants AYA201021097-C03-01, CSD2007-00050, and the Generalitat Valenciana grant PROMETEO-2009-103. The calculations have been performed on the Lluís Vives cluster of the University of Valencia.

\section{References}

[1] Berger E. et al., GCN 11854 (2011), 1

[2] Levan A. J. et al., GCN 11886 (2011), 1
[3] Fruchter A. et al., GCN 11881 (2011), 1

[4] Cenko, B. S. et al., ApJ 753 (2012), 77

[5] Bloom J. et al., Science 333 (2011), 203

[6] Levan A. J. et al., Science 333 (2011), 199

[7] Giannios D., Metzger B. D., MNRAS 416 (2011), 2102

[8] Rees M. J., Nature 333 (1988), 523

[9] Metzger B. D., Giannios D., Mimica P. MNRAS 420 (2012), 3528

[10] Berger E. et al., ApJ 748 (2012), 36

[11] Sari R., Piran T. ApJ 455 (1995), L143

[12] Mimica P., Aloy M. A., Mueller E. A\&A 466 (2007), 93

[13] Mimica P., Giannios D., Aloy M.A. A\&A 494 (2009), 879

[14] Mimica P. et al., ApJ 696 (2009), 1142

[15] Tabik S. et al., Proceedings of the High Performance Computing (HIPC) 2011, (IEEEXplore, 2011), 1-10

[16] Tabik S. et al., Computer Physics Communication 193 (2012), 1937

[17] Mimica P, Giannios D., Aloy M. A., MNRAS 407 (2010), 2501 